\title{
Wavelength-Selective Reflector Based on a Circular Array of Coupled Microring Resonators
}

\author{
Joyce K. S. Poon, Student Member, IEEE, Jacob Scheuer, Member, IEEE, and Amnon Yariv, Life Fellow, IEEE
}

\begin{abstract}
We propose and analyze a novel type of wavelengthselective reflector for planar lightwave technology based on a circular array of coupled microring resonators. Narrow-band reflection peaks can be achieved without the need for additional complex lithography and processing steps. The ring resonators also allow for simple and wide-range tuning of the reflection peak.
\end{abstract}

Index Terms-Integrated optics, microresonators, optical filters, tunable filters.

\section{INTRODUCTION}

$\mathbf{S}$ EMICONDUCTOR lasers that exhibit stable wavelengths and narrow bandwidths are key elements in modern optical communication systems [1]. For wavelength-division-multiplexing applications, it is also desired that the laser's wavelength could be dynamically tuned. In most lasers, stable lasing wavelength is achieved by employing either distributed Bragg reflectors (DBRs) [2] or external cavities [3]-[5]. However, the use of DBR or external cavities requires additional processing steps that complicate the fabrication of the laser structures. In addition, tuning the reflection wavelength of a DBR over a wide wavelength range without deteriorating the reflection characteristics is not trivial and requires careful design and implementation.

In this letter, we present a new type of in-plane wavelengthselective reflector which is based on coupled microring resonators. In the lateral coupling scheme, the ring resonators allow for the fabrication of a reflector in a single lithography step. Moreover, it is possible to dynamically tune the reflection peak simply by changing the effective index of the rings using, for example, the thermooptic or electrooptic effect [6], [7]. While incorporating microrings as mode filters in semiconductor lasers with reflective end facets was recently proposed and analyzed [8]-[10], the use of ring resonators as reflectors has not been previously suggested or studied.

Our device consists of a circular array of $N>2$ ring resonators coupled to a waveguide, as shown in Fig. 1. In a laser, the waveguide will be fabricated from an optically active material, and the other end of the waveguide can be coupled to a similar reflector or can consist simply of a reflective facet. The circular array can also be regarded as a "super" ring resonator formed by a microring coupled resonator optical waveguide [11]-[13]. A wave propagating in the waveguide excites a travelling wave inside the ring resonator array. From Fig. 1, we observe that for an odd number of rings $(N \geq 3)$, the device may act as a re-

Manuscript received November 5, 2003; revised December 12, 2003.

The authors are with the Electrical Engineering Department and the Applied Physics Department, California Institute of Technology, Pasadena, CA 91125 USA (e-mail: poon@caltech.edu).

Digital Object Identifier 10.1109/LPT.2004.826152

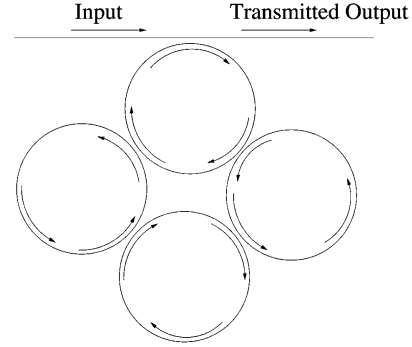

(a)

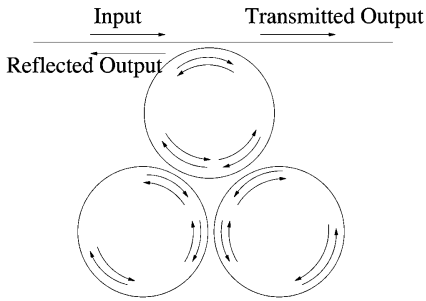

(b)
Fig. 1. Schematic of the reflector. (a) For an even number of rings, the device is always transmitting. (b) For an odd number of rings, the device can be reflecting.

TABLE I

LIST OF SYMBOLS

\begin{tabular}{cc}
\hline Symbol & Significance \\
\hline$a_{n}, b_{n}, c_{n}^{\prime}, d_{n}^{\prime}$ & counter-clockwise propagating fields \\
$c_{n}, d_{n}, a_{n}^{\prime}, b_{n}^{\prime}$ & clockwise propagating fields \\
$x_{n}$ & field vector $[a, b, c, d]_{n}$ \\
$\kappa$ & coupling coefficient \\
$t$ & transmission coefficient \\
$\beta$ & propagation constant \\
$\alpha$ & gain or loss, imaginary part of $\beta$ \\
$\theta$ & angle of an external vertex of an equilateral polygon \\
\hline
\end{tabular}

flector, but for an even number of rings $(N \geq 4)$, the device is always nonreflecting.

\section{TRANSFER MATRIX ANALYSIS}

To analyze light propagation in the resonator array, we use a transfer matrix formalism. The symbols used in this letter are summarized in Table I. In the transfer matrix method, one matrix describes the coupling of light between resonators and another describes the wave propagation inside the resonators [13]-[16]. The forward and backward propagating field components are defined in Fig. 2. We use the vector $x_{n}$ to represent the field components in the $n-1$ th ring

$$
x_{n} \equiv\left[\begin{array}{llll}
a & b & c & d
\end{array}\right]_{n}^{T}
$$

To describe the coupling of waves between adjacent resonators, we assume that the coupling length is much greater than the wavelength of light such that only the phase-matched waves are coupled. Hence, there is no mixing between the clockwise and counterclockwise propagating waves, and the coupling can be represented by a $4 \times 4$ matrix

$$
\begin{aligned}
x_{n+1} & =\left[\begin{array}{cc}
P & 0 \\
0 & P
\end{array}\right] x_{n}^{\prime} \equiv \mathbb{P} x_{n}^{\prime} \\
P & =\frac{1}{\kappa}\left[\begin{array}{cc}
-t & 1 \\
-1 & t^{*}
\end{array}\right], \quad n \geq 0
\end{aligned}
$$




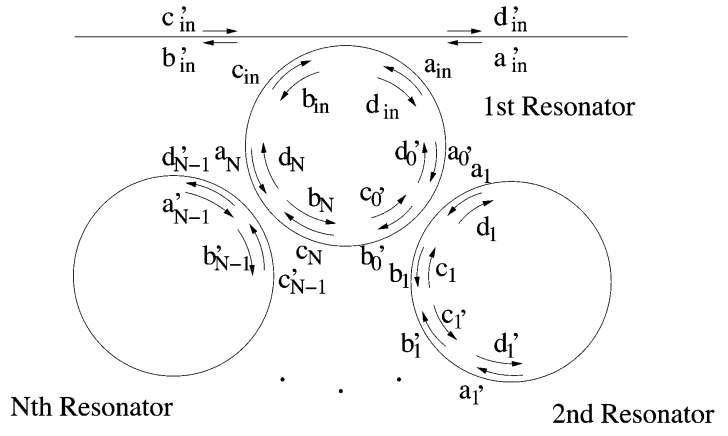

Fig. 2. Circular array of an arbitrary number of rings with the clockwise and counterclockwise fields labeled.

where $\kappa$ and $t$ are the dimensionless coupling and transmission coefficients. $P$ is unitary such that $|\kappa|^{2}+|t|^{2}=1$.

We can relate $x_{n}^{\prime}$ and $x_{n}$ with a propagation matrix such that

$$
\begin{aligned}
x_{n}^{\prime} & =\left[\begin{array}{cc}
0 & Q \\
Q^{\dagger} & 0
\end{array}\right] x_{n} \equiv \mathbb{Q} x_{n} \\
Q & =\left[\begin{array}{cc}
0 & e^{-i \beta R \theta} \\
e^{i \beta R(2 \pi-\theta)} & 0
\end{array}\right]
\end{aligned}
$$

where $\beta$ is the propagation constant, $R$ is the radius of the rings, $Q^{\dagger}$ is the conjugate transpose of $Q$, and $\theta$ is determined from the internal angles of the polygon whose vertices are located at the centers of the rings. $\beta$ may be complex, such that $\beta=$ $n_{\mathrm{eff}} \omega / c+i \alpha$, to account for loss or gain. For an equilateral polygon, $\theta$ is

$$
\theta=2 \pi-\frac{\pi(N-2)}{N} .
$$

Combining (2) and (3)

$$
x_{n+1}=\left[\begin{array}{cc}
0 & P Q \\
P Q^{\dagger} & 0
\end{array}\right] x_{n} \equiv \mathbb{T} x_{n} .
$$

For $N(N>2)$ ring resonators in the circular chain, we cascade the matrices to obtain

$$
x_{N}=\mathbb{T}^{N-1} \mathbb{P} x_{0}^{\prime} .
$$

Our goal is to find an expression that depends solely on $x_{\mathrm{in}}^{\prime}$, since the components of $x_{\text {in }}^{\prime}$ will give the transfer functions of the structure. Thus, we seek to manipulate (6) into the form

$$
x_{\mathrm{in}}^{\prime}=B x_{\mathrm{in}}^{\prime}
$$

where $B$ is a matrix to be determined. Equation (7) also has the form of an eigenvalue problem with an eigenvalue of one.

To begin, we note that at the first resonator, the coupling to the external waveguide is

$$
x_{\mathrm{in}}=\mathbb{P}_{\mathrm{in}} x_{\mathrm{in}}^{\prime}
$$

Moreover, there are six phase relations in the first resonator

$$
\begin{aligned}
a_{0}^{\prime} & =d_{\mathrm{in}} e^{-i \beta R \theta / 2}, \quad d_{0}^{\prime}=a_{\mathrm{in}} e^{i \beta R \theta / 2} \\
b_{0}^{\prime} & =c_{N} e^{i \beta R(2 \pi-\theta)}, \quad c_{0}^{\prime}=b_{N} e^{-i \beta R(2 \pi-\theta)} \\
a_{N} & =b_{\mathrm{in}} e^{-i \beta R \theta / 2}, \quad d_{N}=c_{\mathrm{in}} e^{i \beta R \theta / 2} .
\end{aligned}
$$

We use (9) to express $x_{N}$ and $x_{0}^{\prime}$ in terms of elements in $x_{\text {in }}$ in (6). $b_{N}$ and $c_{N}$ simplify to

$$
\begin{aligned}
& \left.\begin{array}{rl}
b_{N} & =\frac{a_{\mathrm{in}} A_{24} e^{i \beta R \theta / 2}}{1-A_{23} e^{-i \beta R(2 \pi-\theta)}} \\
c_{N} & =\frac{d_{\mathrm{in}} A_{31} e^{-i \beta R \theta / 2}}{1-A_{32} e^{i \beta R(2 \pi-\theta)}}
\end{array}\right\} N=\text { even } \\
& \left.\begin{array}{l}
b_{N}=\frac{d_{\mathrm{in}} A_{21} e^{-i \beta R \theta / 2}+a_{\mathrm{in}} A_{22} A_{34} e^{i \beta R(2 \pi+\theta / 2)}}{1-A_{22} A_{33}} \\
c_{N}=\frac{a_{\mathrm{in}} A_{34} e^{i \beta R \theta / 2}+d_{\mathrm{in}} A_{21} A_{33} e^{-i \beta R(2 \pi-\theta / 2)}}{1-A_{22} A_{33}}
\end{array}\right\} N=\text { odd }
\end{aligned}
$$

where $A_{i j}$ is the $i j$ th element of $A$ and $A \equiv \mathbb{T}^{N-1} \mathbb{P}$. Hence, invoking (8), we can rewrite (6) as

$$
M \mathbb{P}_{\text {in }} x_{\text {in }}^{\prime}=\mathbb{T}^{N-1} \mathbb{P} W \mathbb{P}_{\text {in }} x_{\text {in }}^{\prime}
$$

where $M$ and $W$ express $b_{N}, c_{N}, b_{0}^{\prime}$, and $c_{0}^{\prime}$ using (9) and (10). Finally, we can rewrite (11) in our desired form (7)

$$
x_{\mathrm{in}}^{\prime}=\mathbb{P}_{\mathrm{in}}^{-1} M^{-1} \mathbb{T}^{N-1} \mathbb{P} W \mathbb{P}_{\mathrm{in}} x_{\mathrm{in}}^{\prime} \equiv B x_{\mathrm{in}}^{\prime} .
$$

However, $\operatorname{Det}(W)=0$, rendering $B$ noninvertible. This is expected because physically the system is fully characterized relative to a single input, so the four components of $x_{\text {in }}^{\prime}$ are not linearly independent variables. Thus, we have some freedom in selecting the form of the eigenvector $x_{\text {in }}^{\prime}$. Assuming only a single input, we set one of the inputs to the circular chain of rings to zero, say $a_{\text {in }}^{\prime}$, and we take the transmission and reflectance relative to $c_{\text {in. }}^{\prime}$. The resultant eigenvector has the form

$$
x_{\text {in }}^{\prime}=\left[\begin{array}{llll}
0 & b_{\text {in }}^{\prime} & 1 & d_{\text {in }}^{\prime}
\end{array}\right]^{T}
$$

where $d_{\text {in }}^{\prime}$ is the transmission function and $b_{\text {in }}^{\prime}$ is the reflection function. They can be calculated by solving the matrix equation

$$
\left[\begin{array}{cc}
\frac{B_{42}}{1-B_{44}} & -1 \\
1 & -\frac{B_{24}}{1-B_{22}}
\end{array}\right]\left[\begin{array}{l}
b_{\text {in }}^{\prime} \\
d_{\text {in }}^{\prime}
\end{array}\right]=\left[\begin{array}{c}
\frac{B_{43}}{1-B_{44}} \\
\frac{B_{23}}{1-B_{22}}
\end{array}\right]
$$

where $B_{i j}$ is the $i j$ th element of $B$. The solution also satisfies

$$
\begin{aligned}
& B_{32} b_{\text {in }}^{\prime}+B_{33}+B_{34} d_{\text {in }}^{\prime}=1 \\
& B_{12} b_{\text {in }}^{\prime}+B_{13}+B_{14} d_{\text {in }}^{\prime}=0
\end{aligned}
$$

ensuring that it is self-consistent with (12).

\section{RESULTS AND DISCUSSION}

We use (14) to compute the reflectance and transmittance spectra of the circular array-based reflector. For an even number of rings, the structure is verified to be purely transmitting. For lossless rings, the even number of rings acts as an all-pass filter. Fig. 3(a) and (b) shows the transmission and phase characteristics of an array of four resonators. The interresonator coupling is $\kappa=-0.5 i$ and the coupling between the waveguide and the array is also $\kappa_{\mathrm{in}}=-0.5 i$. The radius of the rings is $100 \mu \mathrm{m}$ and their effective index is 1.5 . The transmission drops and the phase changes most rapidly at the resonances of the "super" resonator. The phase response is not strongly dependent on loss.

Fig. 3(c) and (d) shows the spectra of three coupled resonators for various losses. The interresonator coupling is $\kappa=-0.08 i$ and the coupling between the waveguide and the array is $\kappa_{\mathrm{in}}=$ 


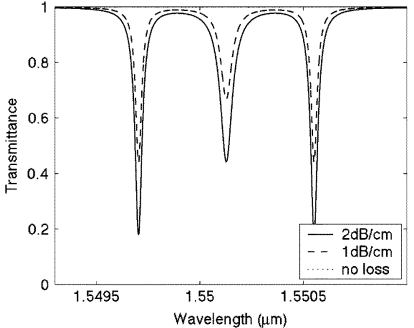

(a)

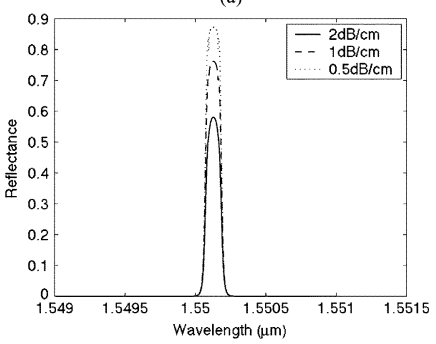

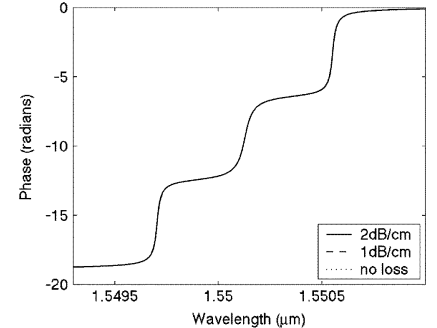

(b)

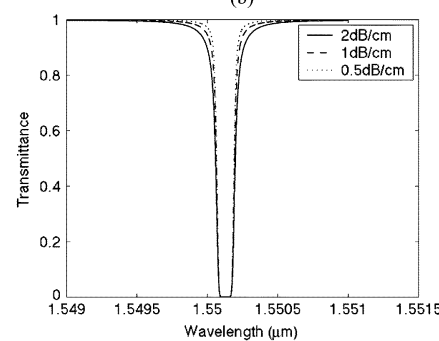

(d)
Fig. 3. Top: (a) Transmittance and (b) phase response of an array of four resonators for various losses. $\kappa=-0.5 i, \kappa_{\mathrm{in}}=-0.5 i$. Bottom: (c) Reflectance and (d) transmittance of an array of three resonators for various losses. $\kappa=-0.08 i, \kappa_{\text {in }}=-0.53 i$. For both cases, $r=100 \mu \mathrm{m}$ and $n_{\text {eff }}=1.5$.

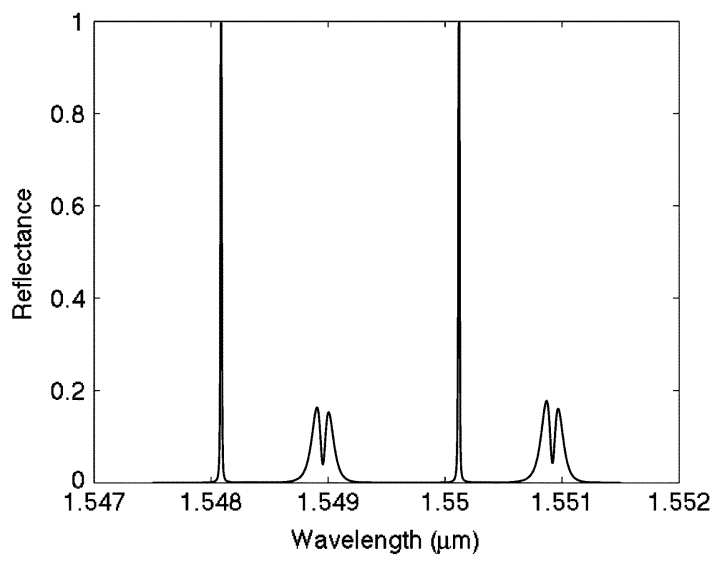

Fig. 4. Reflectance from an array consisting of three different resonators. $r_{1,3}=130 \mu \mathrm{m}, r_{2}=125 \mu \mathrm{m}, \kappa=-0.3 i, \kappa_{\text {in }}=-0.85 i$.

$-0.53 i$. The radius of the rings is $100 \mu \mathrm{m}$ and their effective index is 1.5. The structure exhibits a narrow reflection peak centered at $1.55 \mu \mathrm{m}$ and its free spectral range is $2.4 \mathrm{~nm}$. The maximum reflectance achievable is inversely proportional to the propagation loss in the rings. In general, to obtain narrow reflection peaks, weak interresonator coupling is required. For filtering applications, the input coupling can be chosen to optimize the flatness of the transmission and reflection spectra.

An advantage to the matrix formalism is that it can readily deal with an array composed of an arbitrary mix of resonators and coupling constants simply by accounting for the differences in resonator sizes, coupling, and internal angles in the transfer matrices. By varying the resonators, we can more finely tune the strongly reflected frequencies. Fig. 4 shows the reflectance spectrum for an array of three resonators in which the second resonator is of a different size. The rings are lossless with interresonator coupling of $-0.3 i$ and the coupling to the waveguide is $-0.85 i$. The first and third rings (using the notation in Fig. 2) have a radius of $r_{1,3}=130 \mu \mathrm{m}$, while the second ring has a radius of $r_{2}=125 \mu \mathrm{m}$. The main reflection peaks are spaced $2 \mathrm{~nm}$ apart. However, even though the coupling coefficients are higher than the previous example, the reflection peaks are narrower than the structure composed of identical resonators with a smaller coupling strength [as in Fig. 3(c)]. The use of different resonators provides an additional degree of freedom to obtain narrow reflection peaks.

\section{CONCLUSION}

A circular array of ring resonators, when coupled to a waveguide, offers a new alternative to a wavelength-selective reflector for planar lightwave circuits. The width of the reflection peaks can be narrowed by decreasing interresonator coupling or by using resonators of varying sizes. The advantage of this reflector is that it can be readily fabricated in an integrated optical circuit without any additional processing steps. Moreover, the reflector can be made tunable by using the thermal-optic or electrooptic effect. The device can be used as a laser end-mirror, where the tunable and wavelength-selective nature of the reflector can offer a stabilized yet tunable output wavelength.

\section{REFERENCES}

[1] G. P. Agrawal, Fiber-Optic Communication Systems. New York: Wiley, 1997.

[2] A. Yariv, Optical Electronics in Modern Communications. New York: Wiley, 1997.

[3] T. Schrans, S. Sanders, and A. Yariv, "Broad-band wavelength tunable picosecond pulses from $\mathrm{CW}$ passively mode-locked two-section multiple quantum-well lasers," IEEE Photon. Technol. Lett., vol. 4, pp. 323-326, Apr. 1992.

[4] J.-I. Hashimoto, T. Takagi, T. Kato, G. Sasaki, M. Shigehara, K. Murashima, M. Shiozaki, and T. Iwashima, "Fiber-Bragg-grating external cavity semicondutor laser (FGL) module for DWDM," $J$. Lightwave Technol., vol. 21, pp. 2002-2009, Sept. 2003.

[5] K. Vahala, K. Kyuma, A. Yariv, S.-K. Kwong, M. Cronin-Golomb, and K. Y. Lau, "Narrow linedwidth, single frequency semiconductor laser with a phase conjugate external cavity mirror," Appl. Phys. Lett., vol. 49, no. 23, pp. 1563-1565, 1986.

[6] P. Rabiei, W. H. Steier, C. Zhang, and L. R. Dalton, "Polymer micro-ring filters and modulators," J. Lightwave Technol., vol. 20, pp. 1968-1975, Nov. 2002.

[7] P. Rabiei and W. H. Steier, "Tunable polymer double micro-ring filters," IEEE Photon. Technol. Lett., vol. 15, pp. 1255-1257, Sept. 2003.

[8] B. Liu, A. Shakouri, and J. E. Bowers, "Passive microring-resonatorcoupled lasers," Appl. Phys. Lett., vol. 79, no. 22, pp. 3561-3563, 2001.

[9] —-, "Wide tunable double ring resonator coupled lasers," IEEE Photon. Technol. Lett., vol. 14, pp. 600-602, May 2002.

[10] Z. Bian, B. Liu, and A. Shakouri, "In-P passive ring-resonator-coupled lasers," IEEE J. Quantum Electron., vol. 39, pp. 859-865, July 2003.

[11] A. Yariv, Y. Xu, R. K. Lee, and A. Scherer, "Coupled-resonator optical waveguide: A proposal and analysis," Opt. Lett., vol. 24, no. 11, pp. 711-713, 1999.

[12] G. T. Paloczi, Y. Huang, A. Yariv, and S. Mookherjea, "Polymeric MachZehnder interferometer using serially coupled microresonators," Opt. Express, vol. 11, no. 21, pp. 2666-2671, 2003.

[13] J. K. S. Poon, J. Scheuer, S. Mookherjea, G. T. Paloczi, Y. Huang, and A. Yariv, "Matrix analysis of microring coupled-resonator optical waveguides," Opt. Express, vol. 12, no. 1, pp. 90-103, 2004.

[14] K. Oda, N. Takato, and H. Toba, "A wide-FSR waveguide double-ring resonator for optical FDM transmission systems," J. Lightwave Technol., vol. 9, pp. 728-736, June 1991.

[15] R. Orta, P. Savi, R. Tascone, and D. Trinchero, "Synthesis of multiple-ring resonator filters for optical systems," IEEE Photon. Technol. Lett., vol. 7, pp. 1447-1449, Dec. 1995.

[16] G. Griffel, "Synthesis of optical filters using ring resonator arrays," IEEE Photon. Technol. Lett., vol. 12, pp. 810-812, July 2000. 